\title{
COMPACT CONFORMALLY FLAT HYPERSURFACES
}

\author{
BY
}

\author{
MANFREDO DO CARMO, MARCOS DAJCZER AND FRANCESCO MERCURI
}

\begin{abstract}
Roughly speaking, a conformal space is a differentiable manifold $M^{\prime \prime}$ in which the notion of angle of tangent vectors at a point $p \in M^{n}$ makes sense and varies differentiably with $p$; two such spaces are (locally) equivalent if they are related by an angle-preserving (local) diffeomorphism. A conformally flat space is a conformal space locally equivalent to the euclidean space $R^{n}$. A submanifold of a conformally flat space is said to be conformally flat if so its induced conformal structure; in particular, if the codimension is one, it is called a conformally flat hypersurface.

The aim of this paper is to give a description of compact conformally flat hypersurfaces of a conformally flat space. For simplicity, assume the ambient space to be $R^{n+1}$. Then, if $n \geqslant 4$, a conformally flat hypersurface $M^{n} \subset R^{n+1}$ can be described as follows. Diffeomorphically, $M^{n}$ is a sphere $S^{n}$ with $b_{1}(M)$ handles attached, where $b_{1}(M)$ is the first Betti number of $M$. Geometrically, it is made up by (perhaps infinitely many) nonumbilic submanifolds of $R^{n+1}$ that are foliated by complete round $(n-1)$-spheres and are joined through their boundaries to the following three types of umbilic submanifolds of $R^{n+1}$ : (a) an open piece of an $n$-sphere or an $n$-plane bounded by round $(n-1)$-sphere, (b) a round $(n-1)$-sphere, (c) a point.
\end{abstract}

\section{Introduction.}

1.1. The aim of this paper is to present a rather complete description of compact conformally flat hypersurfaces of a simply-connected, $(n+1)$-dimensional space form, for $n \geqslant 4$. Before stating our results, we will recall some known facts and definitions, mainly in order to fix our notation.

Manifolds are $C^{\infty}$ and boundaryless. A Riemannian manifold $M^{n}$ is (locally) conformally flat if for each point $p \in M^{n}$ there exists an open neighborhood $V$ of $p$ in $M$ and a conformal diffeomorphism of $V$ onto an open set of $R^{n}$ (superscripts will denote dimensions and will be dropped when clear from the context). An immersion $x: M^{n} \rightarrow \bar{M}^{n+k}$ of a differentiable manifold into a Riemannian manifold $\bar{M}^{n+k}$ is a conformally flat immersion if $M^{n}$ is conformally flat with respect to the induced metric; if $k=1$, we will say that $x$ is a conformally flat hypersurface.

1.2. The following notation will be used throughout the paper.

Let $M^{n}$ be a differentiable manifold and $x: M^{n} \rightarrow \bar{M}^{n+k}$ an immersion into a Riemannian manifold $\bar{M}^{n+k}$. We will denote by $\bar{\nabla}$ the Riemannian connection on $\bar{M}$ and by $\nabla$ the induced connection on $M$. Let $X$ and $Y$ be local tangent vector

Received by the editors December 12, 1983 and, in revised form, April 13, 1984.

1980 Mathematics Subject Classification. Primary 53A30; Secondary 53C40. 
fields along $x$; then

$$
\alpha(X, Y)=\bar{\nabla}_{X} Y-\nabla_{X} Y
$$

will denote the second fundamental form of $x$. By fixing a point $p \in M$ and a local unit normal vector field $N$ along $x$ in a neighborhood of $p, \alpha$ determines a selfadjoint linear map $A_{N}: T_{p} M \rightarrow T_{p} M$ of the tangent space $T_{p} M$ of $M$ at $p$, namely,

$$
\left\langle A_{N}(X), Y\right\rangle=\langle\alpha(X, Y), N\rangle \text {. }
$$

We will call $A_{N}$ the Weingarten operator of $x$ relative to $N$. When $k=1$, we will call $N$ a local orientation at $p$. Given a local orientation $N$, we will denote by $\lambda_{1}, \ldots, \lambda_{n}$ the eigenvalues of $A_{N}$. We will use $M(c)$ to denote a Riemannian manifold with constant curvature $c$; when such a manifold is, in addition, complete and simplyconnected, it will be denoted by $\tilde{M}(c)$.

For $n \geqslant 4$ it is well known that $x: M^{n} \rightarrow M^{n+1}(c)$ is conformally flat if and only if, after a possible re-enumeration, $\lambda_{1}=\lambda_{2}=\cdots=\lambda_{n-1}$ (see Cartan [1] or, for a simple proof, see e.g. [7]); notice that this condition does not depend on the choice of a local orientation. For convenience, we will set $\lambda_{1}=\lambda, \lambda_{n}=\mu$ and will denote by $U=\{p \in M ; \lambda=\mu\}$ the set of umbilic points of $M$. We will denote by $(a, b)$ an open interval of the real line.

1.3. In $\$ 2$ we will prove our first main result which describes the geometric structure of a compact conformally flat hypersurface $x: M^{n} \rightarrow \tilde{M}^{n+1}(c), n \geqslant 4$. To simplify the statement, let us call a compact submanifold $\Sigma^{k} \subset M^{n}$ a $k$-sphere if the restriction $x \mid \Sigma^{k}: \Sigma^{k} \rightarrow \tilde{M}^{n+1}(c)$ is a totally umbilic immersion.

1.4 THEOREM. Let $x: M^{n} \rightarrow \tilde{M}^{n+1}(c)$ be an immersed conformally flat hypersurface, $n \geqslant 4, M^{n}$ compact and connected. Assume that $U \neq \varnothing$. Then:

(i) Each connected component $D$ of the set of nonumbilic points admits a codimension one foliation by $(n-1)$-spheres; in particular $D$ is diffeomorphic to $S^{n-1} \times(a, b)$. Furthermore, the boundary of $D$ has at most two connected components and each such component is either a point, an $(n-1)$-sphere or two $(n-1)$-spheres with a common point.

(ii) Each connected component of $U$ is a point, an $(n-1)$-sphere, or an n-dimensional umbilic set bounded by points or by a union of $(n-1)$-spheres such that two such spheres have at most a common point.

A more precise description of the possible shapes of the boundary of $D$ is as follows (see Figure 1): It consists of two points (the closure $\bar{D}$ of $D$ is then diffeomorphic to an $n$-sphere, Figure 1(a)), or one round $(n-1)$-sphere $(\bar{D}$ is diffeomorphic to $S^{n-1} \times S^{1}$-Figure 1(b)-or to a generalized Klein bottle), or one point and one round $(n-1)$-sphere ( $\bar{D}$ is diffeomorphic to an $n$-ball, Figure $1(\mathrm{~d}))$, or two round $(n-1)$-spheres that either are disjoint (Figure 1(e)) or have one common point (Figure 1(c)). Thus we can think of a compact conformally flat hypersurface as nonumbilic "conformally flat handles" that are foliated by $(n-1)$ spheres and are used to join points, $(n-1)$-spheres, and pieces of umbilic $n$-submanifolds of $\tilde{M}(c)$. A typical picture is given in Figure 2 . 

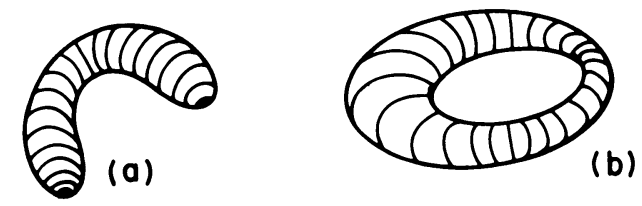

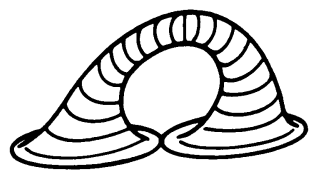

(c)

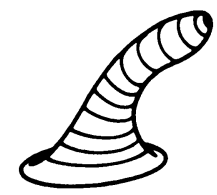

(d)

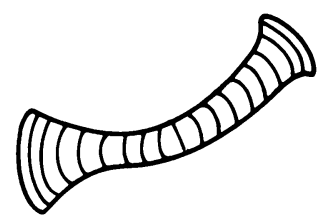

(e)

Figure 1. Possible shapes of a connected component of $M-U, U \neq \varnothing$.

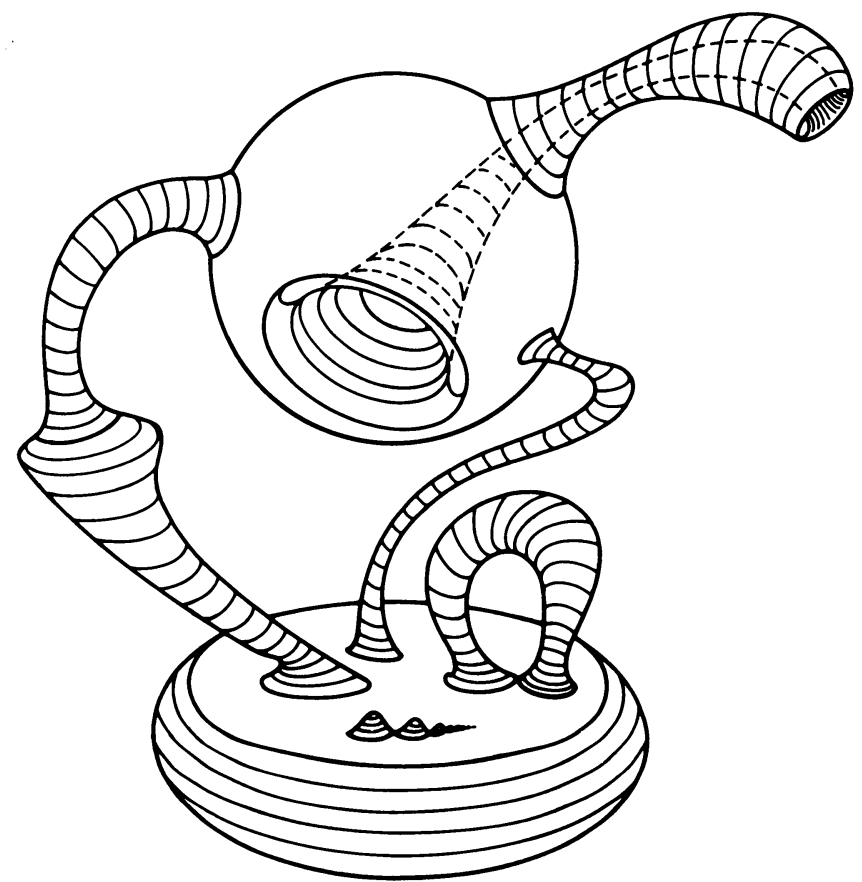

FIGURE 2. A typical compact conformally flat hypersurface of $R^{n+1}, n \geqslant 4$.

1.5 REMARK. Since metric completeness is not a conformal invariant, compactness is the simplest global condition that can be imposed on a conformally flat manifold. A notion of "conformal completeness" was considered in Kuiper [5] but it turned out to be equivalent to compactness.

1.6 Remark. Some attempts towards a classification of conformally flat hypersurfaces have appeared in the literature: [6, 7], and [3, Theorem 4.4, p. 165]. They are, however, incomplete.

1.7 REMARK. Theorem 1.4 confirms essentially a conjecture by N. Kuiper (personal communication). We want to thank him for discussions that lead us to a better understanding of the subject. 
1.8. In $\$ 3$ we describe a method (essentially, although not explicitly, given in E. Cartan [1]; cf. Remark 3.20) that yields a large number of examples of conformally flat hypersurfaces. This is again used in $\$ 4$ to show that we can perform "conformal surgeries" in the nonumbilic "conformally flat handles" that appear in (i) of Theorem 1.4 (cf. Figure 2). By a conformal surgery we mean that we cut off a small nonumbilic "conformally flat handle" and fill in the resulting spherical holes in such a way that the hypersurface thus obtained is still conformally flat. In $\S 5$, the conformal surgery is used to prove our second main result, namely a necessary and sufficient condition for a compact orientable differentiable manifold to admit immersion as a conformally flat hypersurface of $\tilde{M}^{n+1}(c), n \geqslant 4$ (see Theorem 1.10).

1.9. For later use, we describe here the construction of attaching a handle to a differentiable manifold. Let $r B^{n} \subset R^{n}$ denote an open ball of radius $r$ in $R^{n}$ and identify $B^{n}-\frac{1}{3} \bar{B}^{n} \approx\left(\frac{2}{3}, 1\right) \times S^{n-1}$; here $\bar{B}^{n}$ denotes the closure of $B^{n}$. Let $f_{1}, f_{2}$ : $B^{n} \rightarrow M^{n}$ be two embeddings (if $M^{n}$ is oriented, one is orientation-preserving and the other orientation-reversing; otherwise, they are both orientation-preserving) with $f_{1}\left(B^{n}\right) \cap f_{2}\left(B^{n}\right)=\varnothing$. Let $M_{f}, f=\left(f_{1}, f_{2}\right)$, be obtained from

$$
M-\left\{f_{1}\left(\frac{1}{3} B^{n}\right) \cup f_{2}\left(\frac{1}{3} B^{n}\right)\right\}
$$

by the identification

$$
f_{1}(t x)=f_{2}((1-t) x), \quad t \in\left(\frac{1}{3}, \frac{2}{3}\right), x \in S^{n-1} .
$$

It is well known that if $g=\left(g_{1}, g_{2}\right)$ is another pair of embeddings as above, $M_{g}$ is diffeomorphic to $M_{f}$. We will say that $M_{f}$ is obtained from $M$ by attaching $a$ handle. We will denote by $S_{b}^{n}$ a manifold obtained from the standard sphere $S^{n}$ by attaching $b$ handles.

1.10 THEOREM. Let $M^{n}$ be a compact, connected, differentiable manifold of dimension $n \geqslant 4$. Then $M^{n}$ can be immersed as a conformally flat hypersurface $x: M^{n} \rightarrow$ $\tilde{M}^{n+1}(c)$ if and only if $M$ is diffeomorphic to $S_{b}^{n}$.

1.11 Remark. A notation of "intrinsic" conformal surgery was introduced by Kulkarni [6] who was able to show that a connected sum of two conformally flat manifolds can be defined to be conformally flat.

1.12 Remark. It should be remarked that Theorem 1.10 is the exact analogue of the classification theorem for compact surfaces (which are, of course, conformally flat). To the best of our knowledge, the case $n=3$ is still open.

1.13 REMARK. A theorem weaker than Theorem 1.10, namely, that if $x: M^{n} \rightarrow$ $\tilde{M}^{n+1}$ is conformally flat, $M^{n}$ compact, $n \geqslant 4$, then $M^{n}$ is homeomorphic to $S_{b}^{n}$ can be proved without conformal surgery, and does not depend on the material of $\S \S 3$ and 4. This will be clear in $\S 5$.

1.14 RemarK. After this paper had been written, we received a manuscript of $U$. Pinkall who has obtained, independently, a result that is essentially equivalent to Theorem 1.10.

We want to thank the referee for valuable comments in the first version of this paper, especially in $\S 3$. 


\section{The geometric structure; proof of Theorem 1.4.}

2.1. Since $x\left(M^{n}\right) \subset \tilde{M}^{n+1}(c)$ is compact, we can by a convenient conformal transformation, assume that $x$ is a conformally flat hypersurface of the euclidean space $R^{n+1}$. We will use this assumption in the rest of the paper.

The following lemma is essentially the first part of (i) of Theorem 1.4.

2.2. LemMA. Let $M^{n}$ be compact, $n \geqslant 4$, and let $x: M^{n} \rightarrow R^{n+1}$ be a conformally flat hypersurface. Then the set $M-U$ of nonumbilic points has a codimension one foliation so that the image $x(\Sigma) \subset R^{n+1}$ of each leaf $\Sigma$ is a complete $(n-1)$-sphere; in particular, an open connected component of $M-U$ is diffeomorphic to $S^{n-1} \times(a, b)$.

Proof. Choose a local orientation, say $N$, around each point of $M-U$. Let $D_{\lambda}$ (resp. $D_{\mu}$ ) be the distributions defined by taking at each point of $M-U$ the eigenspace of $A_{N}$ corresponding to the eigenvalue $\lambda$ (resp. $\mu$ ); these distributions do not depend on the local orientation and are thus defined in the entire $M-U$. It is well known [9, p. 372] that such distributions are differentiable, involutive, and that the eigenvalue $\lambda$ is constant along each leaf $\Sigma_{\lambda}$ of $D_{\lambda}$.

From the above we see that local coordinates $\left(u_{1}, \ldots, u_{n-1}, t\right)$ can be so chosen around $p$ that the coordinate vectors $\partial / \partial u_{i}=V_{i}, \partial / \partial t=T$ satisfy

$$
A_{N}\left(V_{i}\right)=\lambda V_{i}=-\bar{\nabla}_{V_{i}} N, \quad A_{N}(T)=\mu T=-\bar{\nabla}_{T} N,
$$

where $\bar{\nabla}$ denotes the canonical connection of $R^{n+1}$. Thus, since $R^{n+1}$ is flat,

$$
0=-\bar{\nabla}_{T} \bar{\nabla}_{V_{i}} N+\bar{\nabla}_{V_{i}} \bar{\nabla}_{T} N=(\lambda-\mu) \bar{\nabla}_{T} V_{i}+T(\lambda) V_{i}-V_{i}(\mu) T,
$$

hence, since $\lambda \neq \mu$,

$$
\bar{\nabla}_{T} V_{i}=-\frac{\lambda^{\prime}}{\lambda-\mu} V_{i}+\frac{V_{i}(\mu)}{\lambda-\mu} T
$$

here and elsewhere, a prime denotes derivative in $t$.

Now consider a leaf $\Sigma_{\lambda} \subset M$ and denote by $i: \Sigma_{\lambda} \rightarrow M$ its inclusion map. Then, by setting $\|T\|=1 / \alpha$, and denoting by $\tilde{A}_{N}$ and $\tilde{A}_{\alpha T}$ the Weingarten operators of $x \circ i$ relative to $N$ and $\alpha T$, respectively, we obtain

$$
\tilde{A}_{N}\left(V_{i}\right)=\lambda V_{i}, \quad \tilde{A}_{\alpha T}\left(V_{i}\right)=\frac{\alpha \lambda^{\prime}}{\lambda-\mu} V_{i} .
$$

It follows from (2.3) that the leaf $\Sigma_{\lambda}$ is umbilic relative to the immersion $x \circ i$ : $\Sigma_{\lambda} \rightarrow R^{n+1}$. Thus we can find an orthonormal pair of vectors $\zeta, \zeta^{\perp}$, in the plane $\{T, N\}$, such that the corresponding Weingarten operators of $x \circ i: \Sigma_{\lambda} \rightarrow R^{n+1}$ satisfy

$$
\tilde{A}_{\zeta^{\perp}}\left(V_{i}\right)=0, \quad \tilde{A_{\zeta}}\left(V_{i}\right)=\beta V_{i}, \quad i=1, \ldots, n-1,
$$

where $\beta^{2}$ is the (constant) sectional curvature of $\Sigma_{\lambda}$. In fact, it is easily checked that, if $\beta \neq 0$,

$$
\begin{gathered}
\zeta=\left(\frac{\lambda^{\prime} \alpha}{\lambda-\mu} \alpha T+\lambda N\right) / \beta, \quad \zeta^{\perp}=\left(\lambda \alpha T-\frac{\lambda^{\prime} \alpha}{\lambda-\mu} N\right) / \beta \\
\beta^{2}=\frac{\lambda^{\prime 2} \alpha^{2}}{(\lambda-\mu)^{2}}+\lambda^{2}
\end{gathered}
$$


Thus through each point of $M-U$ there passes a leaf $\Sigma_{\lambda}$, where $x\left(\Sigma_{\lambda}\right) \subset R^{n+1}$ is either a piece of an $(n-1)$-plane (if $\beta=0$ ) or of an $(n-1)$-sphere (if $\beta \neq 0$ ). By a theorem of Reckziegel [8, Theorem 1 (iv)], a limit point of a geodesic of $\Sigma_{\lambda}$ still belongs to $M-U$. Thus the completion of $\Sigma_{\lambda}$ is contained in $M-U$. Since $M$ is compact, $x\left(\Sigma_{\lambda}\right)$ is an $(n-1)$-sphere, i.e., $\beta \neq 0$. This completes the proof.

2.6 Corollary of THE Lemma. A compact conformally flat hypersurface $x: M^{n} \rightarrow$ $R^{n+1}$ without umbilic points is either homeomorphic to $S^{n-1} \times S^{1}$ (if $M$ is orientable) or to a "generalized Klein bottle" (if $M$ is nonorientable).

This follows from the classification of sphere bundles over $S^{1}$ (cf. Steenrod [10, p. 134]).

The following lemma proves the second part of Theorem 1.4(i).

2.7 Lemma. Let $U \neq \varnothing$ and let $D \subset M$ be a connected component of $M-U$. Then the boundary $\mathrm{Bd} D$ of $D$ has at most two connected components and each such component is either a point, an $(n-1)$-sphere, or the union of two $(n-1)$-spheres with a common point.

Proof. Let $p \in \operatorname{Bd} D$ and let $\left\{p_{n}\right\}$ be a sequence of points with $p_{n} \in D$ and $\left\{p_{n}\right\} \rightarrow p$. Since $p_{n} \in M-U$, there exists, by Lemma 2.2, an $(n-1)$-sphere $\Sigma_{n} \subset M$ passing through $p_{n}$. We can choose the sequence $\left\{p_{n}\right\}$ in such a way that $\Sigma_{n} \neq \Sigma_{m}$, for $m \neq n$. We can also assume that, for all $n, p_{n} \in V$, where $V \subset M$ is a neighborhood of $p$ such that the restriction $x \mid V$ is an embedding.

Let us denote by $T_{n}$ the tangent space of $x\left(\Sigma_{n}\right)$ at $p_{n}$, by $R_{n}$ the radius of $x\left(\Sigma_{n}\right)$ and by $\xi_{n}$ a unit normal vector to $x\left(\Sigma_{n}\right) \subset R^{n+1}$ at $p_{n}$ in the affine hyperplane of $R^{n+1}$ containing $x\left(\Sigma_{n}\right)$. Since $M$ is compact, $\left\{R_{n}\right\}$ is a bounded sequence. Again, by an argument of compactness, there exists a subsequence $\left\{n_{k}\right\}$ of $\{n\}$ such that the subsequences $\left\{R_{n_{h}}\right\},\left\{T_{n_{h}}\right\}$ and $\left\{\xi_{n_{h}}\right\}$ converge to, say, $R, T$ and $\xi$, respectively. It follows that a subsequence $\left\{\Sigma_{n_{h}}\right\}$ of $\left\{\Sigma_{n}\right\}$ converges to an $(n-1)$-sphere $\Sigma \subset M$ passing through $p$. Since $p \in U$, the same holds for all points of $\Sigma$, by Lemma 2.2 , and $\Sigma \subset \mathrm{Bd} D$. We notice that if $R=0, \Sigma$ reduces to a single point.

We now observe that $D$ is not compact; otherwise, $D=M$ and $U=\varnothing$, contrary to the hypothesis. Thus, by Lemma $2.2, D$ is homeomorphic to $S^{n-1} \times(-1,1)$, and the homeomorphism maps each slice $S^{n} \times\{t\}, t \in(-1,1)$, onto a leaf $\Sigma_{t}$ of the foliation of $D$. By what we have just proved, $\mathrm{Bd} D$ is the nonvoid union of (perhaps degenerate) $(n-1)$-spheres.

We claim that as $t \nearrow 1, \Sigma_{t}$ converges to a unique (perhaps degenerate) sphere $\Sigma_{1}$. Assume that the contrary holds. Then there exist sequences $\Sigma_{t_{n}}, \Sigma_{t_{m}}^{\prime}$ converging to $\Sigma_{1}$ and $\Sigma_{1}^{\prime}$, respectively, and $\Sigma_{1} \neq \Sigma_{1}^{\prime}$. Let $p \in \Sigma_{1}, p \notin \Sigma_{1}^{\prime}$, and let $V$ be a connected neighborhood of $p$. If $V$ is sufficiently small, $V$ intersects all $\Sigma_{t_{n}}$ and misses all $\Sigma_{t_{m}}^{\prime}$, for $m$ and $n$ sufficiently large. Since each leaf of the foliation disconnects $D, V$ is not connected. This contradiction proves our claim.

It follows that $\mathrm{Bd} D=\Sigma_{1} \cup \Sigma_{-1}$, where $\Sigma_{-1}$ is the unique sphere obtained as $t \searrow-1$. If $\Sigma_{1}$ reduces to a point, this point is an isolated connected component of Bd $D$. This follows from the fact that if a neighborhood $V$ of $p=\Sigma_{1}$ is sufficiently 
small, then, for all $t$ sufficiently near to $1, \Sigma_{t} \subset V$; thus no point $q \in \operatorname{Bd} D$ can be joined to $p$ by a continuous curve of boundary points. The same argument shows that if either $\Sigma_{1}$ or $\Sigma_{-1}$ (or both) are degenerate, then $\Sigma_{1} \cap \Sigma_{-1}=\varnothing$.

Therefore we can assume that both $\Sigma_{1}$ and $\Sigma_{-1}$ are nondegenerate. If they intersect transversally, two leaves of the foliation of $D$ would intersect, which is impossible. Thus, either $\Sigma_{1}$ and $\Sigma_{-1}$ are tangent, in which case $\mathrm{Bd} D=\Sigma_{1} \cup \Sigma_{-1}$, or $\Sigma_{1} \cap \Sigma_{-1}=\varnothing$ in which case $\Sigma_{1}$ and $\Sigma_{-1}$ are the two connected components of Bd D. This completes the proof of Lemma 2.7.

Part (ii) of Theorem 1.4 is proved along the same lines of the above lemma. We omit the details and restrict ourselves to making a couple of observations.

2.8 ReMARK. If $D$ is a connected component of $M-U$ such that the limit spheres $\Sigma_{1}$ and $\Sigma_{-1}$ are nondegenerate and $\Sigma_{1}=\Sigma_{-1}$, then $M=\bar{D}$ and, exactly as in Corollary 2.6, $M$ is homeomorphic to either $S^{n-1} \times S^{1}$ (in the orientable case), or to a "generalized Klein bottle" (in the nonorientable case).

2.9 REMARK. The tangent spheres that make up a connected component of $\operatorname{Bd} U$ may belong to the boundaries of distinct components of $M-U$.

\section{Nonumbilic conformally flat hypersurfaces.}

3.1. Let $x: M^{n} \rightarrow R^{n+1}, n \geqslant 4$, be a conformally flat hypersurface that contains no umbilics and let $p \in M$. As we saw in the proof of Lemma 2.2, we have in a neighborhood $W$ of $p$ a coordinate system $\left(u_{1}, \ldots, u_{n-1}, t\right)$ adapted to the distributions $D_{\lambda}, D_{\mu}$ and a local orientation $N$. Let $V_{i}=\partial / \partial u_{i}, T=\partial / \partial t$ be the coordinate fields in $W$ and choose the local orientation $N$ so that $\left\langle A_{N} V_{i}, V_{i}\right\rangle=\lambda\left\|V_{i}\right\|^{2} \leqslant 0$. Now assume that $\lambda \neq 0$, set $c(u, t)=x+\lambda^{-1} N$ and observe that $\partial c / \partial u_{i}=0$, $i=1, \ldots, n-1$. This means that $c=c(t)$ depends on $t$ alone. Since the same holds for $\lambda$, we obtain a curve $c(t)$ and a one-parameter family of $n$-spheres $\Sigma_{t}^{n}$ given by $|y-c(t)|^{2}=\lambda^{-2}$ such that, for each $t$, the sphere $\Sigma_{t}^{n}$ is tangent to the hypersurface $x(M)$. Thus the hypersurface $x$ is locally the envelope of a one-parameter family of spheres with centers $c(t)$ and radii $-\lambda^{-1}(t)$. Notice that if $\lambda=0$, the sphere of the family becomes a tangent hyperplane (which, from the viewpoint of conformal geometry, is indistinguishable from a sphere), and we can say that a nonumbilic conformally flat hypersurface is locally the envelope of a one-parameter family of umbilics.

Now suppose that at a point $p \in M$ we have $\lambda=0$. Let $H$ be the tangent hyperplane of $x(M)$ at $x(p)$ and choose a point $q \in R^{n+1}$ so that $q \notin H, q \notin x(M)$. By making an inversion $\tau$ in $R^{n+1}$ with center $q$, we obtain that $\tau(H)$ is a sphere, hence the value of $\lambda$ at $p$ in the transformed (nonumbilic) hypersurface $\tau \circ x$ : $M^{n} \rightarrow R^{n+1}$ is nonzero. Thus we can assume that, modulo a conformal transformation of the ambient space, $\lambda$ is nonzero in a neighborhood of $p$.

The nonumbilic conformally flat hypersurfaces can be explicitly given by the following construction which merely describes an envelope of a one-parameter family of spheres.

3.2. Let $c:(a, b) \subseteq R \rightarrow R^{n+1}$ be an immersed curve and let $r:(a, b) \rightarrow R$ be a positive real differentiable function with $\left|r^{\prime}(t)\right|<\left\|c^{\prime}(t)\right\|, t \in(a, b)$. Set

$$
S=r r^{\prime} /\|c\|^{2}, \quad R=r\left(1-\left(\left(r^{\prime}\right)^{2} /\left\|c^{\prime}\right\|^{2}\right)\right)^{1 / 2} \text {. }
$$


For each $t$, consider the affine hyperplane of $R^{n+1}$ orthogonal to $c^{\prime}(t)$ and passing through

$$
\gamma(t)=c(t)-S(t) c^{\prime}(t)
$$

in this hyperplane, consider an $(n-1)$-sphere $\Sigma_{t}^{n-1}$ with radius $R(t)$ and center $\gamma(t)$. As $t$ runs in $(a, b), \sum_{t}^{n-1}$ describes a set that is the image set of the map $y$ : $(a, b) \times S^{n-1} \rightarrow R^{n+1}$ given by

$$
y(t, q)=c-S c^{\prime}(t)+R \phi(t, q), \quad t \in(a, b), q \in S^{n-1} .
$$

Here $S^{n-1}$ is a fixed unit $(n-1)$-sphere in a euclidean $n$-space, and $\phi:(a, b) \times$ $S^{n-1} \rightarrow R^{n+1}$ is any differentiable map which, for a fixed $t$, is an immersion $\phi_{t}$ of $S^{n-1}$ in $R^{n+1}$ that satisfies $\left\langle\phi_{t}, c^{\prime}(t)\right\rangle=0, \phi_{t}^{2}=1$. It is clear that for any $\phi$ satisfying the above conditions, the image of $y$ will remain the same.

It is easily seen that if we change the parameter $t$ of the curve $c$ to $\tau=\tau(t)$ (with $d \tau / d t \neq 0$ ), this does not affect either the condition $\left|r^{\prime}\right|<\left\|c^{\prime}\right\|$ or the image set of $y$. We will see in a short while the conditions that $y$ must satisfy to be an immersed hypersurface (an example where $y$ presents singularities is given in Remark 3.19).

3.5 Proposition. Assume that in (3.4) y is an immersed hypersurface. Then $y$ is a conformally flat hypersurface without umbilics, and $\lambda \neq 0$ everywhere. Conversely, any conformally flat hypersurface $x: M^{n} \rightarrow R^{n+1}, n \geqslant 4$, without umbilics and with $\lambda \neq 0$ everywhere is locally of the form $y$; furthermore, if $M$ is orientable, $x(M)$ is contained in the image of a hypersurface of the form $y$.

Proof. We will first prove the converse. By the considerations in the beginning of this section, we obtain in a neighborhood $W$ of a point $p \in M$ a curve $c(t)=x+$ $\lambda^{-1} N$ and a positive function $r=-\lambda^{-1}$. By differentiating $c=x+\lambda^{-1} N$ in $t$, we obtain

$$
\begin{aligned}
c^{\prime} & =\frac{\partial x}{\partial t}+\frac{1}{\lambda} \frac{d N}{d t}-\frac{\lambda^{\prime}}{\lambda^{2}} N=T-\frac{1}{\lambda} \mu T-\frac{\lambda^{\prime}}{\lambda^{2}} N \\
& =\frac{\lambda(\lambda-\mu) T-\lambda^{\prime} N}{\lambda^{2}},
\end{aligned}
$$

since $\partial x / \partial t=T$ and $d N / d t=\mu T$. Thus, by setting $\alpha=\|T\|^{-1}$, we conclude that

$$
\left\|c^{\prime}\right\|^{2}=(\lambda-\mu)^{2} / \alpha^{2} \lambda^{2}+\left(r^{\prime}\right)^{2},
$$

hence, since $\lambda-\mu \neq 0,\left|c^{\prime}\right|^{2}>\left(r^{\prime}\right)^{2}$. Thus the curve $c$ and the positive function $r$ satisfy the required condition.

Next, we observe that the compactness of $M$ was used in the proof of Lemma 2.2 only to guarantee that $\beta \neq 0$, where $\beta^{2}$ is the curvature of the leaves of the foliation of $M$ by the distribution $D_{\lambda}$. Since in the present case $\lambda \neq 0$, we can use the same argument as in Lemma 2.2 to conclude that $M$ is foliated by (perhaps incomplete) spheres $\Sigma$ with radii

$$
\left(\frac{\alpha^{2}\left(\lambda^{\prime}\right)^{2}}{(\lambda-\mu)^{2}}+\lambda^{2}\right)^{-1 / 2}=r\left(1-\frac{r^{\prime 2}}{\left\|c^{\prime}\right\|^{2}}\right)^{1 / 2} .
$$


Now, notice that $c^{\prime}$ belongs to the plane spanned by $T$ and $N$, and because of (2.4), $\left\langle c^{\prime}, \zeta\right\rangle=0$. By the above equation, the distance from $c(t)$ to the center of $\Sigma$ is $r r^{\prime} /\left\|c^{\prime}\right\|^{2}$. It follows that the restriction $x \mid W$ is obtained from $c(t)$ and $r(t)$ exactly as in (3.4). Thus $x$ is locally of the form $y$.

Notice that if $M$ is orientable, the curve $c$ and the function $r>0$ are defined for the entire $M$. By taking the arc length as a parameter for $c$, we obtain a hypersurface $y$ such that, locally, the image of $x$ is contained in the image of $y$. Thus the same is true globally, and this completes the first part of the proof.

Now assume that $y$ is an immersed hypersurface. Since $\left\langle\phi, c^{\prime}\right\rangle=0$, we obtain $\|y-c\|^{2}=r^{2}$. Choose orthogonal coordinates $\left(u_{1}, \ldots, u_{n-1}\right)$ for $S^{n-1}$ and differentiate $\|y-c\|^{2}=r^{2}$ in $t$ and in $u_{i}, i=1, \ldots, n-1$, to obtain

$$
\left\langle\partial y / \partial u_{i}, y-c\right\rangle=0, \quad\left\langle\partial y / \partial t-c^{\prime}, y-c\right\rangle=r r^{\prime}
$$

From the above and the fact that $\left\langle y-c, c^{\prime}\right\rangle=-r r^{\prime}$, we obtain that $\langle\partial y / \partial t, y-c\rangle$ $=0$. It follows that $N$ given by

$$
y-c=-r N
$$

is a unit normal vector to $y$. Since $\partial y / \partial u_{i}=-r \partial N / \partial u_{i}$, we conclude that $y$ is a conformally flat hypersurface with $\lambda=-1 / r \neq 0$ as the eigenvalue of $A_{N}$ with multiplicity at least $n-1$. It remains to prove that $y$ is nonumbilic.

To see this we observe that the image of $y$ is naturally foliated by spheres; thus we can choose locally an orthogonal coordinate system $\left(u_{1}, \ldots, u_{n-1}, \tau\right)$ for $y$ adapted to this foliation. By what we have seen in the first part of the proof, this gives rise to a curve $c(\tau)=y+\lambda^{-1} N$, which by (3.8) agrees with $c(t)$. Thus $\left(u_{1}, \ldots, u_{n-1}, t\right)$ are orthogonal coordinates, and by (3.7) we have that $\lambda \neq \mu$, since $\left\|c^{\prime}\right\|^{2}>(r)^{2}$. This completes the proof of Proposition 3.5.

3.9. Given a nonumbilic conformally flat immersion, it will be convenient to say that $M$ is saturated by the foliation $D_{\lambda}$ if together with $p \in M$ it contains the completion of the leaf passing through $p$.

3.10 Corollary. Let $x: M^{n} \rightarrow R^{n+1}, n \geqslant 4$, be a nonumbilic conformally flat immersion and assume that $M$ is saturated by the foliation $D_{\lambda}$. Let $H$ be a saturated neighborhood of a leaf of $D_{\lambda}$ and assume that $\lambda \neq 0$ and that $H$ is homeomorphic to $S^{n-1} \times(a, b)$. Then there exits an immersion $y: H \rightarrow R^{n+1}$ of the form (3.4) such that $x(H)=y(H)$.

This follows from the fact that $H$ is simply-connected, hence orientable.

3.11. Now we want to determine the singular points of $y$. Let $(t, q) \in(a, b) \times$ $S^{n-1}$, and choose orthogonal coordinates $\left(u_{1}, \ldots, u_{n-1}\right)$ around $q \in S^{n-1}$. As we have seen in the proof of the last proposition,

$$
\left\langle\partial y / \partial u_{i}, y-c\right\rangle=0, \quad\langle\partial y / \partial t, y-c\rangle=0, \quad y-c \neq 0 .
$$

Thus $(t, q)$ is a singular point for $y$ if and only if $\partial y / \partial t=0$. The conditions below can be checked to be independent of the parametrization of $c$, and describe therefore singularities of the image of $y$. 
3.12 Proposition. Let $y$ be given as in (3.4). A point $(t, q)$ is singular for $y$ if and only if both conditions below are satisfied:

$$
\begin{aligned}
& \text { (i) } 1-S^{\prime}=\frac{1}{\left\|c^{\prime}\right\|^{2}}\left\{R\left\langle\phi, c^{\prime \prime}\right\rangle+S\left\langle c^{\prime}, c^{\prime \prime}\right\rangle\right\}, \\
& \text { (ii) } R^{\prime}=S\left\langle c^{\prime \prime}, \phi\right\rangle .
\end{aligned}
$$

Condition (i) implies condition (ii), and if $S \neq 0$ (i.e. if $r^{\prime} \neq 0$ ), condition (ii) implies condition (i). Furthermore (i) can be written as

$$
\left\|c^{\prime}\right\|^{2}-r^{\prime 2}-r r^{\prime \prime}+S\left\langle c^{\prime}, c^{\prime \prime}\right\rangle=R\left\langle\phi, c^{\prime \prime}\right\rangle .
$$

Proof. We use the coordinates $\left(u_{1}, \ldots, u_{n-1}, t\right)$ introduced in the proof of Proposition 3.5 and notice that they are orthogonal parameters for $y$. Then

$$
y^{\prime}=c^{\prime}-S^{\prime} c^{\prime}-S c^{\prime \prime}+R^{\prime} \phi+R \phi^{\prime}=\left(1-S^{\prime}\right) c^{\prime}-S c^{\prime \prime}+R \phi^{\prime}+R^{\prime} \phi .
$$

Since $\left\langle\partial y / \partial u_{i}, y\right\rangle=0$, we obtain $\left\langle\partial \phi / \partial u_{i}, y^{\prime}\right\rangle=0$, hence

$$
0=\left\langle y^{\prime}, \partial \phi / \partial u_{i}\right\rangle=-S\left\langle c^{\prime \prime}, \partial \phi / \partial u_{i}\right\rangle+R\left\langle\partial \phi / \partial u_{i}, \phi^{\prime}\right\rangle, \quad i=1, \ldots, n-1,
$$

since $\left\langle\partial \phi / \partial u_{i}, c^{\prime}\right\rangle=0,\left\langle\partial \phi / \partial u_{i}, \phi\right\rangle=0$. It follows from (3.14) that $R \phi^{\prime}-S c^{\prime \prime}$ is orthogonal to $\partial \phi / \partial u_{i}$ for all $i$. Thus $R \phi^{\prime}-S c^{\prime \prime}$ belongs to the plane generated by $\phi$ and $c^{\prime}$ and it is easily computed that

$$
R \phi^{\prime}-S c^{\prime \prime}=\left\langle-R \phi-S c^{\prime}, c^{\prime \prime} /\left\|c^{\prime}\right\|\right\rangle c^{\prime} /\left\|c^{\prime}\right\|-S\left\langle c^{\prime \prime}, \phi\right\rangle \phi .
$$

It follows that

$$
y^{\prime}=\left(1-S^{\prime}-R\left\langle\phi, c^{\prime \prime}\right\rangle /\left\|c^{\prime}\right\|^{2}-S\left\langle c^{\prime}, c^{\prime \prime}\right\rangle /\left\|c^{\prime}\right\|^{2}\right) c^{\prime}+\left(R^{\prime}-S\left\langle c^{\prime \prime}, \phi\right\rangle\right) \phi,
$$

and this shows that $y^{\prime}=0$ if and only if both (i) and (ii) hold.

Now a simple computation shows that the derivatives of $R$ and $S$ satisfy

$$
\begin{gathered}
R^{\prime}=S\left(\left\|c^{\prime}\right\|^{2}-r^{\prime 2}-r r^{\prime \prime}+S\left\langle c^{\prime}, c^{\prime \prime}\right\rangle\right) R^{-1}, \\
1-S^{\prime}=\frac{\left\|c^{\prime}\right\|^{2}-r^{\prime 2}-r r^{\prime \prime}}{\left\|c^{\prime}\right\|^{2}}+2\left\langle c^{\prime}, c^{\prime \prime}\right\rangle \frac{S}{\left\|c^{\prime}\right\|^{2}} .
\end{gathered}
$$

Thus

$$
R R^{\prime}=S\left\{\left(1-S^{\prime}\right)\left\|c^{\prime}\right\|^{2}-\left\langle c^{\prime}, c^{\prime \prime}\right\rangle S\right\} .
$$

It follows that if (i) holds, then $R R^{\prime}=S R\left\langle\phi, c^{\prime \prime}\right\rangle$, that is, (ii) holds. Furthermore, if $S \neq 0$ and (ii) holds, then (i) also holds. Finally, we obtain from (3.16) that (i) can be written as

$$
\left\|c^{\prime}\right\|^{2}-r^{\prime 2}-r r^{\prime \prime}+S\left\langle c^{\prime}, c^{\prime \prime}\right\rangle=R\left\langle\phi, c^{\prime \prime}\right\rangle
$$

We leave it to the reader to check that conditions (i) and (ii) are independent of the parametrization of the curve $c(t)$. This completes the proof of Proposition 3.12.

3.17 Remark. The first part of Proposition 3.5 together with Proposition 3.12 can be used to construct a large number of examples of conformally flat hypersurfaces. For instance, if $c$ is a straight line, so is the curve $\gamma(t)=c-S c^{\prime}$ and we obtain a 
rotation hypersurface. On the other hand, if $r$ is a small constant, then $c(t)=\gamma(t)$ and we obtain a tube around $c$, i.e., the total space of a normal sphere bundle of $c$ with radius $r$. We can also obtain conformally flat hypersurfaces that are neither tubes nor rotation hypersurfaces, as the example below shows.

3.18 EXAMPLE. Let $\left(x_{1}, \ldots, x_{n}\right)$ be cartesian coordinates in $R^{n+1}$. In the plane $x_{1} x_{2}$ choose a circle $c(s)$ centered at the origin and with radius $L>1$. Assume that $\left\|c^{\prime}\right\|=1$ and let $r: \mathbf{R} \rightarrow \mathbf{R}$ be given by

$$
r(s)=\beta^{-1} \sin (s / L)+1,
$$

where $\beta>1$ is a real number. It is easily checked that if $L$ is sufficiently large, the hypersurface given by $y$ in (3.4) is an embedded compact conformally flat hypersurface without umbilics. A restricted class of examples of conformally flat hypersurfaces were found in $[6,7,2]$.

3.19 REMARK. A simple example where $y$ presents a singular set is given by a nonumbilic rotation hypersurface with $r(t)$ chosen in such a way that $R^{\prime}$ changes sign along a leaf where $r^{\prime} \neq 0$. Then $y$ looks like two rotation hypersurfaces meeting along an " $S^{n-1}$-edge".

3.20 Remark. Proposition 3.5 is essentially contained in Cartan [1, p. 88], where he proves with his own methods that a conformally flat hypersurface of $\tilde{M}^{n+1}(c)$ is locally an envelope of a one-parameter family of umbilic submanifolds of $\tilde{M}^{n+1}(c)$. Cartan only consider such envelopes that are immersed hypersurfaces and does not go into the question of singularities treated in Proposition 3.12. Thus he does not describe explicitly the above method of construction of conformally flat hypersurfaces.

\section{Conformal surgery.}

4.1. Let $x: M^{n} \rightarrow R^{n+1}$ be a compact conformally flat hypersurface and let $p \in M$ be a nonumbilic point with $\lambda(p) \neq 0$. By Corollary 3.10 the image of a small saturated neighborhood $H$ of the spherical leaf that passes through $p$ can be written as $y(H)$, where $y: H \rightarrow R^{n+1}$ is in the form (3.4). We will say that $H$ is a conformal handle around $p$. The aim of this section is to show that we can cut off a small conformal handle $H^{\prime} \subset H$ around $p$ and fill in the resulting spherical holes in such a way that we still obtain a conformally flat hypersurface. This process will be called a conformal surgery along the leaf of $p$.

Let $H$ be a conformal handle and assume that the curve $c(t)$ in the expression (3.4) is parametrized proportionally to the arc length, i.e., $\left\|c^{\prime}(t)\right\|=$ const. Let $p$ belong to the leaf corresponding to $t=t_{0}$ and let us omit from $H$ the handle corresponding to the interval $\left(t_{0}-2 \varepsilon, t_{0}+2 \varepsilon\right)$, where $\varepsilon>0$ is small. In $\left(t_{0}-2 \varepsilon, t_{0}\right.$ $-\varepsilon)$, keep the values of $r(t)$ and $\left\|c^{\prime}(t)\right\|$ and change the curve $c$ so that it becomes a straight line in the interval $\left(t_{0}-\varepsilon, t_{0}\right)$. This can be done without introducing singularities, since the condition (3.13) becomes

$$
\left\|c^{\prime}\right\|^{2}-r^{\prime 2}-r r^{\prime \prime}=R\left\langle\phi, c^{\prime \prime}\right\rangle
$$

and the process can be made so that the second member goes monotonically to zero. 
We want to show that we can let $R$ approach zero as $t$ approaches $t_{0}$ without introducing singularities and keeping it conformally flat. Since $y$ is a rotation hypersurface near $t_{0}$, we must make sure, in addition, that $R^{\prime}$ approaches $-\infty$ as $t \rightarrow t_{0}$. This will be taken care of by the following.

4.2 LemmA. As $t \rightarrow t_{0}$, let $\left\|c^{\prime}\right\| \rightarrow \alpha>0,\left\|c^{\prime}\right\|^{\prime} \rightarrow \gamma \leqslant 0, r^{\prime} \rightarrow \pm \alpha, r \rightarrow \beta>0$ and $r^{\prime \prime} \rightarrow \delta$. Then $R=r\left(1-r^{\prime 2} /\left\|c^{\prime}\right\|^{2}\right)^{1 / 2} \rightarrow 0$, and

(i) if $r^{\prime} \rightarrow \alpha$ and $-\delta<|\gamma|$, then $R^{\prime} \rightarrow-\infty$,

(ii) if $r^{\prime} \rightarrow-\alpha$ and $\delta<|\gamma|$, then $R^{\prime} \rightarrow-\infty$.

Proof. The first statement is obvious. To prove (i) and (ii), we write $R^{\prime}$ in the form

$$
R^{\prime}=r^{\prime}\left(1-\frac{r^{\prime 2}}{\left\|c^{\prime}\right\|^{2}}\right)-r\left(1-\frac{r^{\prime 2}}{\left\|c^{\prime}\right\|^{2}}\right)^{-1 / 2}\left\{\frac{r^{\prime \prime}}{\left\|c^{\prime}\right\|}-\frac{\left\|c^{\prime}\right\|^{\prime}}{\left\|c^{\prime}\right\|} \frac{r^{\prime}}{\left\|c^{\prime}\right\|}\right\}\left(\frac{r^{\prime}}{\left\|c^{\prime}\right\|}\right)
$$

and notice that, as $t \rightarrow t_{0}$,

$$
R^{\prime} \rightarrow \frac{\beta}{0}\left(\frac{\gamma \pm \delta}{\alpha}\right)
$$

where the minus sign corresponds to the case (i) and the plus sign corresponds to the case (ii). The lemma follows immediately.

4.3 LeMMA. Let $y$ be given in the interval $\left(t_{0}-\varepsilon, t_{0}\right)$ by

$$
y=c(t)-S c^{\prime}(t)+R \phi,
$$

where $c(t)$ is a straight line with $\left\|c^{\prime}(t)\right\|=$ const. Then we can change $r(t)$ and $\left\|c^{\prime}(t)\right\|$ so that $R \rightarrow 0$ as $t \rightarrow t_{0}$, the resulting hypersurface is conformally flat, and no singularities are introduced.

Proof. The delicate point is to control the occurrence of singularities that, in this case, are determined by

$$
\left\|c^{\prime}\right\|^{2}-r^{\prime 2}-r r^{\prime \prime}+\frac{r r^{\prime}}{\left\|c^{\prime}\right\|}\left\|c^{\prime}\right\|=0
$$

Notice that as long as we keep $\left\|c^{\prime}\right\|=$ const, the above condition reduces to

$$
A=\left\|c^{\prime}\right\|^{2}-r^{\prime 2}-r r^{\prime \prime}=0 .
$$

The proof will follow by analyzing various cases (see Figure 3):

Case 1. $r^{\prime}>0, r^{\prime \prime}>0,\left\|c^{\prime}\right\|^{2}-r^{\prime 2}>r r^{\prime \prime}$. Thus $A>0$.

We will derive the interval $\left(t_{0}-\varepsilon, t_{0}\right)$ into four (not necessarily equal) parts: I, II, III and IV.

In I we decrease monotonically $\left(r^{\prime}\right)^{2}$ and $r^{\prime \prime}$ so that $r^{\prime \prime}=0$ at the right endpoint of I. Clearly $A>0$ throughout I. By (4.5), no singularities are introduced.

In II we decrease $\left(r^{\prime}\right)^{2}$ until it becomes zero, keep $r^{\prime \prime}$ and $r^{\prime \prime \prime}$ negative, and let $r^{\prime \prime \prime} \rightarrow 0$ at the right endpoint of II. Notice that

$$
A^{\prime}=-3 r^{\prime} r^{\prime \prime}-r r^{\prime \prime \prime} \text {. }
$$




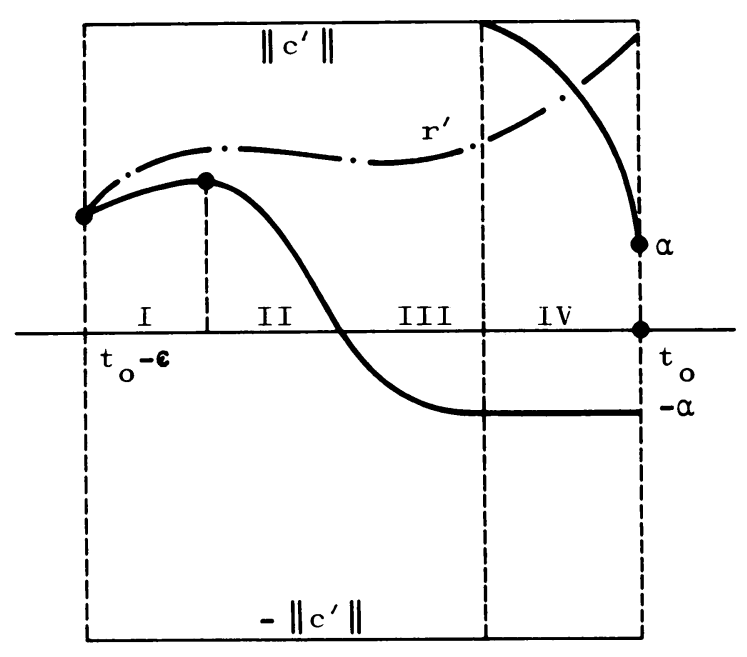

FIGURE 3

It follows that $A^{\prime}>0$, hence $A$ is positive in II and no singularities appear. Notice also that at the right endpoint of II, we have that $A=\left\|c^{\prime}\right\|^{2}+r\left|r^{\prime \prime}\right|$.

In III we change $r^{\prime}$ from zero to a constant negative value by keeping $r^{\prime \prime}<0$ and changing the sign of $r^{\prime \prime \prime}$ to $r^{\prime \prime \prime}>0$. By (4.6), we see that $A$ increases in III from $A=\left\|c^{\prime}\right\|^{2}+r\left|r^{\prime \prime}\right|>0$ to $A=\left\|c^{\prime}\right\|^{2}-\left(r^{\prime}\right)^{2}>0$. Thus $A>0$ throughout the interval III, and no singularities appear.

So far we have kept $\left\|c^{\prime}\right\|=$ const. In IV, however, we keep $r^{\prime}=$ const $=-\alpha$ and let $\left\|c^{\prime}\right\|$ approach $\alpha$ as $t \rightarrow t_{0}$. By Lemma 4.2 we see that no singularity will appear at $t=t_{0}$. For the rest of the interval, we observe that, since $r^{\prime \prime}=0 \mathrm{in} \mathrm{IV,} \mathrm{we} \mathrm{must}$ show that

$$
\left\|c^{\prime}\right\|^{2}-r^{\prime 2}+\frac{r r^{\prime}}{\left\|c^{\prime}\right\|}\left\|c^{\prime}\right\|^{\prime}=A
$$

does not vanish in IV. Notice that in a small interval around the left endpoint of IV, $A>0$. A computation gives that

$$
A^{\prime}=\left(\frac{2\left\|c^{\prime}\right\|^{2}+r^{\prime 2}}{\left\|c^{\prime}\right\|}\right)\left\|c^{\prime}\right\|^{\prime}-\frac{r r^{\prime}}{\left\|c^{\prime}\right\|^{2}}\|c\|^{\prime 2}+\frac{r r^{\prime}}{\left\|c^{\prime}\right\|}\left\|c^{\prime}\right\|^{\prime \prime},
$$

where we can assume that $\left\|c^{\prime}\right\|^{\prime \prime}<0$ and $\left\|c^{\prime}\right\|^{\prime}<0$. By adjusting the length of IV, we can make $\left\|c^{\prime}\right\|^{\prime}$ sufficiently large so that the absolute value of the second term of the above sum is larger than the first term. Since $r^{\prime}<0, A^{\prime}$ will then be positive, hence $A$ will not vanish in IV.

Case 2. $r^{\prime}>0, r^{\prime \prime}>0,\left\|c^{\prime}\right\|^{2}-r^{2}<r r^{\prime \prime}$. Thus $A<0$.

Assume that $r^{\prime \prime \prime}>0$. By (4.6) we see that $A^{\prime}<0$. By letting $r^{\prime} \rightarrow\left|c^{\prime}\right|=$ const as $t \rightarrow t_{0}$ and keeping $r^{\prime}, r^{\prime \prime}, r^{\prime \prime \prime}$ positive, we easily see that the lemma holds. If $r^{\prime \prime \prime}<0$, we let $r^{\prime \prime \prime} \rightarrow 0$ by changing very little $r^{\prime}$ and $r^{\prime \prime}$ (so that $A$ remains negative); we then change the sign of $r^{\prime \prime \prime}$ and proceed as above.

All the other cases are similar (or can be reduced) to the previous cases, and we leave them to the reader. This concludes the proof of Lemma 4.3. 
4.7 Proposition. Let $x: M^{n} \rightarrow R^{n+1}, n \geqslant 4$, be a compact conformally flat hypersurface and let $p \in M$ be a nunumbilic point with $\lambda(p) \neq 0$. Then a conformal surgery can be performed along the leaf of $p$.

This follows immediately from the considerations in the beginning of this section, Lemma 4.2 and Lemma 4.3.

\section{The differentiable structure; proof of Theorem 1.10.}

5.1. Clearly any manifold diffeomorphic to $S_{b}^{n}$ can be immersed as a conformally flat hypersurface of $R^{n+1}$ by joining a convenient number of $n$-spheres in $R^{n+1}$ through general conformal handles.

To prove the converse, let $M^{n}$ be compact, orientable, $n \geqslant 4$, and let $x: M^{n} \rightarrow R^{n+1}$ be a conformally flat hypersurface. Let $\xi \in R^{n+1},\|\xi\|=1$, be a regular value of the Gauss map of $x$ and denote by $h_{\xi}=\langle x, \xi\rangle$ the height function of $x$ relative to $\xi$. At a critical point of $h_{\xi}, \xi$ is normal to $x(M)$ and the second fundamental form of $x$ is the hessian of $h_{\xi}$. Since there exists an eigenvalue $\lambda$ of the second fundamental form with multiplicity at least $n-1, h_{\xi}$ has only critical points of indices $0,1, n-1, n$.

If $M$ is homeomorphic to a sphere, then by a theorem of $\mathrm{N}$. Kuiper [4] the metric induced by the conformally flat immersion $x: M^{n} \rightarrow R^{n+1}$ is conformally equivalent to the standard metric in $S^{n}$. Thus $M^{n}$ is diffeomorphic to a standard $n$-sphere.

A crucial point in the proof of Theorem 1.10 is the following simple observation. Assume that $M$ is not homeomorphic to a sphere. Then there exists at least one critical point $p$ of $h_{\xi}$ with index 1 or $n-1$. Thus $p$ is a nonumbilic point with $\lambda(p) \neq 0$ and there exists a conformal handle around $p$.

Assume that $M$ is not homeomorphic to a sphere and let $p_{1}, \ldots, p_{k}$ be the critical points of $h_{\xi}$ with indices 1 or $n-1$. Let $H_{j}, j=1, \ldots, p$, be a conformal handle around $p_{j}$. By cutting off a sufficiently small handle $H_{j}^{\prime} \subset H_{j}$ around $p_{j}$, we can fill in smoothly the resulting spherical holes in $M$ by $n$-disks in such a way that we elliminate the critical point $p_{j}$ and add perhaps maxima or minima to $h_{\xi}$.

We claim that for at least one $j=1, \ldots, k$, the above process does not disconnect $M$. Otherwise, we would end up with $k+1$ connected components $M_{i}$. Since on each $M_{i}, h_{\xi}$ has only maxima and minima, $M_{i}$ is homeomorphic to a sphere. On the other hand, since each $H_{j}^{\prime}$ disconnects $M$, the two connected components of the boundary of $H_{j}^{\prime}$ are in distinct connected components of $\cup M_{i}$, hence $M$ is a connected sum of spheres, hence homeomorphic to a sphere, a contradiction to the hypothesis.

Now let $H_{j_{1}}, \ldots, h_{j_{h}}$ be the handles whose omission, in a given order, do not disconnect $M$, and apply the above process to such handles. We obtain a manifold $\tilde{M}$ which by the above argument is homeomorphic to a sphere.

We want to show that $\tilde{M}$ admits a conformally flat immersion in $R^{n+1}$. For that, we need the conformal surgery described in $\S 4$. By applying conformal surgeries at the points $p_{j_{1}}, \ldots, p_{j_{b}}$, we obtain a conformally flat immersion $\tilde{x}: \tilde{M} \rightarrow R^{n+1}$. It follows, by Kuiper's theorem quoted above, that $\tilde{M}$ is diffeomorphic to a standard $n$-sphere.

Thus either $M$ is diffeomorphic to $S_{0}^{n}$ or $M$ is obtained from $S_{0}^{n}$ by $b$ steps, each of which starts from the pair of disks we used to fill in the holes in the conformal 
surgery and proceeds in exactly the same way as in the construction of attaching handles. Thus $M$ is diffeomorphic to $S_{b}^{n}$. Since the fundamental group of $S_{b}^{n}$ is a free group with $b$ generators, the number $b$ of handles is the first Betti number of $M$ and does not depend on the above ordering. This completes the proof of Theorem 1.10.

\section{REFERENCES}

1. E. Cartan, La déformation des hypersurfaces dans l'espace conforme réel a $n \geqslant 5$ dimensions, Bull. Soc. Math. France 45 (1917), 57-121.

2. T. Cecil and P. Ryan, Conformal geometry and the cyclides of Dupin, Canad. J. Math. 32 (1980), 767-782.

3. B. Y. Chen, Geometry of submanifolds, M. Dekker, New York, 1973.

4. N. Kuiper, On conformally flat spaces in the large, Ann. of Math. (2) 50 (1949), 916-924.

5. On compact conformally euclidean spaces of dimension > 2, Ann. of Math. (2) 52 (1950), $478-490$

6. R. S. Kulkarni, Conformally flat manifolds, Proc. Nat. Acad. Sci. U.S.A. 69 (1972), 2675-2676.

7. S. Nishikawa and Y. Maeda, Conformally flat hypersurfaces in a conformally flat Riemannian manifold, Tôhoku Math. J. 26 (1974), 159-168.

8. H. Reckziegel, Completeness of curvatures surfaces of an isometric immersion, J. Differential Geom. 14 (1979), 7-20.

9. P. Ryan, Homogeneity and some curvature conditions for hypersurfaces, Tôhoku Math. J. 21 (1969), $363-388$.

10. N. Steenrod, Topology of fiher bundles, Princeton Univ. Press, Princeton, N.J., 1951.

IMPA, Estrada Dona Castorina, 110, Rio de Janeiro, 22460, RJ, Brasil (Current address of M. do Carmo and M. Dajczer)

Instituto de Matemática, UNICAMP, Campinas, 13.100, São Paulo, Brasil (Current address of F. Mercuri) 